\title{
Risk of Malnutrition after Gastrointestinal Cancer Surgery: A Propensity Score Matched Retrospective Cohort Study
}

\author{
Sung-Hoon Yoon, M.D. ${ }^{1}$, Bong-Hyeon Kye, M.D., Ph.D. ${ }^{2}$, Hyung-Jin Kim, M.D. ${ }^{1}$, \\ Kyong-Hwa Jun M.D., Ph.D. ${ }^{1}$, Hyeon-Min Cho, M.D., Ph.D. ${ }^{1}$, \\ Hyung-Min Chin, M.D., Ph.D. ${ }^{1}$ \\ Department of Surgery, ${ }^{1}$ St. Vincent's Hospital and ${ }^{2}$ Seoul St. Mary's Hospital, College of Medicine, The Catholic \\ University of Korea, Seoul, Korea
}

\begin{abstract}
Purpose: Patients with cancers arising from the gastrointestinal tract can suffer from nutritional inadequacies caused by various factors. This study investigated the risk of malnutrition after curative surgery in patients with gastric cancer (GC) or colorectal cancer (CRC) using various preoperative and postoperative nutritional screening tools.

Materials and Methods: In the authors' hospital, 407 patients (206 patients with GC and 201 patients with CRC) underwent surgery between July 2011 and June 2012. The patients from the two groups were matched using the propensity score and then analyzed the nutritional data from 170 patients (85 patients in each group), retrospectively. Results: In both groups, the postoperative nutritional status was impaired significantly compared to the preoperative status. The postoperative risk of undernutrition in CRC patients was significantly lower than that of the GC patients according to the Malnutrition Universal Screening Tool $(P=0.007)$. At the time of hospital discharge after surgery, the incidence of a lower serum albumin level $(P=0.002)$ and more than $5 \%$ weight loss $(P=0.013)$ were higher in the $G C$ group than in the CRC group. A comparison of the postoperative nutritional status among the types of surgery in each group, total gastrectomy in the GC group $(P=0.015)$ and proctectomy with diverting stoma in the $C R C$ group $(P=0.06)$, were related to more than $5 \%$ weight loss.

Conclusion: Gastrointestinal cancer surgery might increase the patients' postoperative risk of malnutrition, particularly in GC surgery. Therefore, consecutive assessments of the nutritional status and appropriate nutritional support are necessary after surgery for GC and CRC. (Surg Metab Nutr 2018;9:16-25)
\end{abstract}

Key Words: Colorectal cancer, Gastric cancer, Postoperative risk of malnutrition

\section{INTRODUCTION}

Malnutrition and weight loss are frequent problems in patients with malignant disease, affecting prognosis, length of hospital stay, quality of life, and survival.[1] Although the prevalence of malnutrition varies among tumor types and sites and the stage of the disease, the prevalence of malnutrition in cancer patients is higher than in general patients because of cancer-specific character- istics and the treatment process.[2] Nutritional status of cancer patients is an important factor related to application to a therapeutic approach such as surgery, chemotherapy, radiation therapy, etc. Specifically, cancers arising from the gastrointestinal tract pose major risks to the patient's nutritional status from the cancer itself, impairment of absorption of nutrients, disruption of the secretion of enzymes, disturbance of the passage of intestinal contents from the tumor, and/or consequences of the

Received June 15, 2018. Accepted June 27, 2018.

Correspondence to: Bong-Hyeon Kye, Department of Surgery, Seoul St. Mary's Hospital, College of Medicine, The Catholic University of Korea, 222 Banpodaero, Seocho-gu, Seoul 06591, Korea

Tel: +82-2-2258-6763, Fax: +82-2-595-2992, E-mail: ggbong@catholic.ac.kr

(C) This is an open access article distributed under the terms of the Creative Commons Attribution Non-Commercial License (http://creativecommons.org/licenses/by-nc/4.0) which permits unrestricted non-commercial use, distribution, and reproduction in any medium, provided the original work is properly cited. 
surgery of the gastrointestinal tract. Because curative surgeries for gastrointestinal cancers include radical lymph node dissection as well as bowel resection and anastomosis, bowel rest for a certain period after surgery is required for patients who undergo curative surgery for gastrointestinal cancer. Also, because of the wider range of cancer surgeries with curative intent, it might be more important to consider a patient's nutritional status and support in these types of surgery.

Many investigators have studied perioperative nutritional support options for patients with gastrointestinal cancer.[3-5] However, most randomized clinical trials in this vein have shown that the degree of malnutrition varies considerably, ranging from no weight loss to weight loss exceeding 10\%, and the results obtained by these studies are different in malnourished and non-malnourished patients.[3-5] Also, most studies do not evaluate the effect of surgery on patient nutritional status. Because most patients with gastrointestinal cancer undergo curative surgery, patient nutritional status both preoperative and postoperative is very important.

Recently, many cancer patients undergoing surgery have been treated with enhanced recovery after surgery (ERAS). The nutritional components of ERAS include avoiding fasting, pre-operative fluid and carbohydrate loading, and recommencement of an oral diet on the first postoperative day.[6] According to the European Society of Parental and Enteral Nutrition (ESPEN) guidelines on nutrition in cancer patients published in 2016, every patient in this ERAS program should be screened for malnutrition and, if deemed at risk, given additional nutritional support.[7] Also, patients undergoing multimodal oncological care are at particular risk of progressive nutritional decline. Because neoadjuvant or adjuvant therapy has been performed in a considerable number of patients with gastrointestinal cancer, it is necessary to evaluate the risk of this progressive nutritional decline. In many fields, surgeons evaluate patient pain, wounds, general appearance, and activity at the time of discharge after surgery. However, most of them lack knowledge on how to evaluate patients' nutritional conditions, including appetite or weight loss, compared to their preoperative condition.

In this study, as the first step to assess the progressive nutritional decline from multimodal oncological care, we investigated the change of the nutritional status before vs. after curative surgery in patients with gastric cancer (GC) or colorectal cancer (CRC).

\section{MATERIALS AND METHODS}

\section{Ethics}

Approval was granted from the review board at our institute (VC16RASI0194) prior to study commencement.

\section{Sample size}

A retrospective hospital chart review was performed of 407 Korean patients (206 patients with GC and 201 patients with $\mathrm{CRC}$ ) who underwent surgery at our Hospital, between July 2011 and August 2012. Of these, 371 patients (195 with gastric cancer and 176 with colorectal cancer) underwent radical surgery with curative intent. Of the 371 patients, propensity scores were calculated from the 318 patients with preoperative (admission) and discharge body weight recorded. The propensity score was calculated by logistic regression analysis using the following covariates: age, sex, and American Society of Anesthesiologist (ASA-REF) score. GC and CRC patients were matched $1: 1$ by a caliper distance of $0.001 \%(20 \%$ of the standard deviation of the propensity score), to minimize selection bias. After matching patients, a total of 170 patients ( 85 patients in each group) were included.

\section{Routine postoperative care}

All operations in this study were performed by a specialized surgeon. The operations for GC were performed by two surgeons specialized in upper gastrointestinal tract surgery, based on the location of the tumor patients underwent a total gastrectomy or a subtotal gastrectomy with Billiroth-I or Billiroth-II reconstruction. The operations for CRC were performed by three colorectal surgeons, based on the location of tumor patients underwent, right hemicolectomy, left hemicolectomy, anterior 
resection, or proctectomy and total mesorectal excision with or without stoma. The following section describes the postoperative care for GC and CRC patients. For total gastrectomy, patients adhered to "Nothing per oral (NPO)" until postoperative day (POD) \#5. After the check for safe anastomosis by gastrografin swallowing test, "sips of water (SOW)" was started. Then, patients started solid diets on POD \#7. For subtotal gastrectomy including Billroth-I and Billroth-II reconstruction, patients started SOW at POD \#3 and sold diet at PDO \#5. In patients with colon cancer surgeries including right and left hemicolectomy and anterior resection and in patients with proctectomy with a diverting stoma for rectal cancer, SOW was started at POD \#2 and solid diet at POD \#4. In patients who underwent proctectomy without diverting stoma, SOW was started at POD \#3 and solid diet at POD \#5. All patients received parenteral nutrition (PN) during the NPO period after surgery; however, PN was discontinued when patients started SOW. Patients were discharged from the hospital when they could consume more than half of the food provided, were drain free, and could ambulate the ward without painkillers.

\section{Defining complications}

All complications occurred during the hospital stay and within 30 days after surgery. Postoperative complications were classified using a therapy-oriented 4-level severity grading system, the Clavien-Dindo classification [8]: Grade I means any deviation from the normal postoperative course without the need for pharmacological treatment or surgical, endoscopic, and radiological interventions (example: atelectasis); Grade II includes complications which require pharmacological treatment, blood transfusions (for the present study, patients were considered to have received a blood transfusion if intraoperative blood loss exceeded $100 \mathrm{~mL}$ and postoperative hemoglobin level was less than $10 \mathrm{~g} / \mathrm{d}$ ), or total parenteral $\mathrm{nu}^{-}$ trition (example: postoperative ileus); Grade III requires surgical, endoscopic or radiological intervention and Grade IV means Life-threatening complication (including CNS complications) requiring IC/ICU management; Grade
$\mathrm{V}$ means death of a patient. Postoperative complications with a Dindo classification 3 or higher were classified as major complications. Patients were divided into two groups according to the presence or absence of morbidity for identifying the association between the preoperative nutritional status and postoperative complications. Patients with Dindo grade I or severe were included to morbidity positive group and patients without any complication to morbidity negative group.

\section{Nutritional risk screening}

Nutrition screening was completed at time of admission for surgery and repeated at discharge. Three nutrition screening tools were utilized: the Nutritional Risk Screening (NRS) 2002,[9] the Malnutrition Universal Screening Tool (MUST),[9] and the Seoul National University Bundang Hospital Nutritional Screening Tool (SNUBH-NST).[10]

The purpose of the MUST system is to detect undernutrition on the basis of association between impaired nutritional status and impaired function. It was primarily developed for use in the community, where serious confounders of the effect of undernutrition are relatively rare.[9] Its practicability has been documented in a number of studies in different community regions in the UK. The purpose of the NRS-2002 system is to detect the presence of undernutrition and the risk of developing undernutrition in the hospital setting. It contains the nutritional components of MUST and severity of disease as a reflection of increased nutritional requirements. It includes four questions as a pre-screening for departments with few at-risk patients.[9]

The SNUBH-NST developed by Seoul National University Bundang Hospital of Korea specifically for the nutritional screening of Koreans.[10] The SNUBH-NST was designed using a nutritional screening index (NSI), which was calculated based on age, body mass index (BMI), serum albumin level, and total lymphocyte count (TLC). According to the tool, age (years) $>65=1, \leq 65=2$; BMI $\left(\mathrm{kg} / \mathrm{m}^{2}\right)<18.5=1, \geq 18.5=2$; serum albumin $(\mathrm{g} / \mathrm{dL})$ $<3.5=1, \geq 3.5=2$; and TLC (cells $\left./ \mathrm{mm}^{3}\right)<900=1, \geq 900=2$ 
can be applied to the formula "NSI=age $\times(1+\mathrm{BMI}) \times 1.5+$ serum albumin $\times(2+\mathrm{TLC}) \times 1.5$." If the result is under 8.75 , the patient is classified as "at risk of malnutrition." This screening tool correlated significantly with age, anthropometrics, laboratory data, and length of stay. Furthermore, the SNUBH-NST was consistent with the NRS 2002 recommended by the European Society for Parenteral and Enteral Nutrition (ESPEN). In a meta-analysis of several studies, the prognostic nutritional index (PNI), which is calculated based on serum albumin and total lymphocyte count, in GC patients has been suggested as a predictive indicator of survival and postoperative complication, and it might be associated with clinicopathological features.[11] The SNUBH-NST was used because it was similar to the PNI.[12]

\section{Data Collection}

All the data were collected through the retrospective chart review. Data collected included patient demographics, operative approach (laparoscopy or open), weight, height, the results from nutritional screening tools at time of admission for surgery and at discharge after surgery, the postoperative morbidity and mortality, the postoperative day starting oral intake other than water (liquid or oral nutritional supplement), and the length of the postoperative hospital stay. Pathologic data from the surgical specimen were reviewed and then classified the pathologic data according to the AJCC $7^{\text {th }}$ staging system in both groups.

\section{Primary and secondary outcomes}

In this study, we primarily intended to compare "the risk of malnutrition' between before and after surgery in patients with GC and CRC with three nutritional screening tools. Additionally, 'the risk of malnutrition' after surgery according to various operation techniques for GC and $\mathrm{CRC}$ respectively were analyzed.

\section{Statistical analyses}

The differences in demographics, pre-operative and post-operative nutritional characteristics (including body weight, BMI, serum albumin level, and total lymphocyte count), and the association among the risk of malnutrition and postoperative morbidity were analyzed between the GC and CRC groups using the $\chi^{2}$ test for categorical variables and the paired t-test for continuous variables and expressed as mean $\pm \mathrm{SD}$. For subgroup analysis according to the type of surgery in GC and CRC groups, continuous variables were compared using one-way ANOVA. And categorical variables were analyzed with the $\chi^{2}$ test. Although significance was defined as $\mathrm{P} \leq 0.05, \mathrm{P} \leq 0.1$ was regarded as marginal significance. All statistical analyses were performed using the Statistical Package of the Social Sciences (SPSS) version 15.0 for Windows (SPSS, Inc., Chicago, IL).

\section{RESULTS}

Propensity score matching resulted in similar age and sex distribution between groups. The preoperative albumin level in the CRC group was higher than that in the GC group ( $4.2 \pm 0.4$ vs. $3.9 \pm 0.5 \mathrm{mg} / \mathrm{dL}, \mathrm{P}=0.010$ ). Patients with preoperative medium or high risk for undernutrition were more in the $\mathrm{CRC}$ group than in the GC group

Table 1. Preoperative patient demographics

\begin{tabular}{|c|c|c|c|c|}
\hline & & $\begin{array}{l}\text { Gastric cancer } \\
\qquad(\mathrm{N}=85)\end{array}$ & $\begin{array}{c}\text { Colorectal } \\
\text { cancer }(\mathrm{N}=85)\end{array}$ & P-value \\
\hline \multirow[t]{2}{*}{ Age (years) } & $\leq 65$ & 49 (57.6\%) & $48(56.5 \%)$ & \\
\hline & $>65$ & 36 (42.4\%) & 37 (43.5\%) & 1.000 \\
\hline \multirow[t]{2}{*}{ Sex } & Male & $52(61.2 \%)$ & $51(60.0 \%)$ & \\
\hline & Female & $33(38.8 \%)$ & $34(40.0 \%)$ & 1.000 \\
\hline \multirow[t]{2}{*}{$\mathrm{BMI}^{\star}\left(\mathrm{kg} / \mathrm{m}^{2}\right)$} & $<18.5$ & $3(3.5 \%)$ & $3(3.5 \%)$ & \\
\hline & $\geq 18.5$ & 82 (96.5\%) & $82(96.5 \%)$ & 1.000 \\
\hline \multirow[t]{2}{*}{$\mathrm{TLC}^{\dagger}$ (cells $/ \mathrm{mm}^{3}$ ) } & $<900$ & $6(7.1 \%)$ & $11(12.9 \%)$ & \\
\hline & $\geq 900$ & 79 (92.9\%) & $74(87.1 \%)$ & 0.307 \\
\hline \multirow[t]{2}{*}{ Albumin (g/dL) } & $<3.5$ & $17(20.5 \%)$ & $5(5.9 \%)$ & \\
\hline & $\geq 3.5$ & $66(79.5 \%)$ & $80(94.1 \%)$ & 0.006 \\
\hline \multirow{2}{*}{$\begin{array}{l}\text { NRS }^{\dagger} 2002 \\
\text { high risk }\end{array}$} & No & $60(70.6 \%)$ & $48(56.5 \%)$ & \\
\hline & Yes & $25(29.4 \%)$ & 37 (43.5\%) & 0.079 \\
\hline \multirow{3}{*}{$\begin{array}{l}\text { Risk of } \\
\text { undernutrition } \\
\text { by } \mathrm{MUST}^{\S}\end{array}$} & Low & 85 (100\%) & $62(72.9 \%)$ & \\
\hline & Medium & 0 & $15(17.6 \%)$ & \\
\hline & High & 0 & $8(9.4 \%)$ & $<0.001$ \\
\hline \multirow{2}{*}{$\begin{array}{l}\text { SNUBH-NST } \\
\text { high risk }\end{array}$} & No & 84 (98.8\%) & 81 (95.3\%) & \\
\hline & Yes & $1(1.2 \%)$ & $4(4.7 \%)$ & 0.368 \\
\hline
\end{tabular}


(27.1\% vs. $0 \%, \mathrm{P}<0.001$ by MUST). According to the NRS 2002, there was a marginal difference in nutritional risk between the two groups $(29.4 \%$ vs. $43.5 \%, \mathrm{P}=0.079)$ (Table 1).

\begin{tabular}{|c|c|c|c|c|}
\hline & & $\begin{array}{l}\text { Gastric } \\
\text { cancer } \\
(\mathrm{N}=85)\end{array}$ & $\begin{array}{c}\text { Colorectal } \\
\text { cancer } \\
(\mathrm{N}=85)\end{array}$ & P-value \\
\hline \multirow[t]{4}{*}{ T stage } & 1 & $54(63.5 \%)$ & 8 (9.4\%) & \\
\hline & 2 & $8(9.4 \%)$ & $13(15.3 \%)$ & \\
\hline & 3 & 10 (11.8\%) & $48(56.5 \%)$ & \\
\hline & 4 & $13(15.3 \%)$ & 16 (18.8\%) & $<0.001$ \\
\hline \multirow{2}{*}{$\begin{array}{l}\text { Lymph node } \\
\text { status }\end{array}$} & Negative & $58(68.2 \%)$ & $44(51.8 \%)$ & \\
\hline & Involved & $27(31.8 \%)$ & $41(48.2 \%)$ & 0.041 \\
\hline \multirow{3}{*}{$\begin{array}{l}\text { Overall TNM } \\
\text { stage }\end{array}$} & I & $57(67.1 \%)$ & $15(17.6 \%)$ & \\
\hline & ॥ & $12(14.1 \%)$ & 29 (34.1\%) & \\
\hline & III & $16(18.8 \%)$ & 41 (48.2\%) & $<0.001$ \\
\hline \multirow[t]{5}{*}{ Morbidity } & No & 80 (94.1\%) & 75 (88.2\%) & \\
\hline & Yes & $5(5.9 \%)$ & $10(11.8 \%)$ & 0.279 \\
\hline & $\begin{array}{l}\text { Anastomosis } \\
\text { problem }\end{array}$ & 2 cases & 2 & 2 \\
\hline & Bleeding & 1 case & 1 & 3 \\
\hline & Ileus & 2 cases & 2 & 5 \\
\hline \multirow{2}{*}{$\begin{array}{r}\text { Clavien-Dindo } \\
\text { classification }\end{array}$} & Below 3 & 83 (97.6\%) & 81 (95.3\%) & 0.682 \\
\hline & 3 or Higher & $2(2.4 \%)$ & $4(4.7 \%)$ & \\
\hline
\end{tabular}

Table 3. Preoperative nutritional state and postoperative morbidity

\begin{tabular}{|c|c|c|c|c|}
\hline & & $\begin{array}{l}\text { Morbidity }(-) \\
(\mathrm{N}=155)\end{array}$ & $\begin{array}{c}\text { Morbidity }(+)^{*} \\
(\mathrm{~N}=15)\end{array}$ & P-value \\
\hline \multirow[t]{2}{*}{ Age (years) } & $\leq 65$ & 66 (90.4\%) & $7(9.6 \%)$ & \\
\hline & $>65$ & 89 (91.8\%) & 8 & 0.790 \\
\hline \multirow[t]{2}{*}{ Sex } & Male & 92 (89.3\%) & $11(10.7 \%)$ & \\
\hline & Female & 63 (94.0\%) & $4(6.0 \%)$ & 0.409 \\
\hline \multirow[t]{2}{*}{$\mathrm{BMI}^{\star}\left(\mathrm{kg} / \mathrm{m}^{2}\right)$} & $<18.5$ & $5(83.3 \%)$ & $1(16.7 \%)$ & \\
\hline & $\geq 18.5$ & 150 (91.5\%) & $14(8.5 \%)$ & 0.430 \\
\hline \multirow[t]{2}{*}{$\mathrm{TLC}^{\dagger}$ (cells $/ \mathrm{mm}^{3}$ ) } & $<900$ & $15(88.2 \%)$ & $2(11.8 \%)$ & \\
\hline & $\geq 900$ & 140 (91.5\%) & 13 (8.5\%) & 0.649 \\
\hline \multirow[t]{2}{*}{ Albumin (g/dL) } & $<3.5$ & 20 (90.9\%) & $2(9.1 \%)$ & \\
\hline & $\geq 3.5$ & 133 (91.1\%) & $13(89.9 \%)$ & 1.000 \\
\hline \multirow{2}{*}{$\begin{array}{l}\text { NRS }^{\dagger} 2002 \\
\text { high risk }\end{array}$} & No & $100(92.6 \%)$ & $8(7.4 \%)$ & \\
\hline & Yes & $55(88.7 \%)$ & $7(11.3 \%)$ & 0.410 \\
\hline \multirow{3}{*}{$\begin{array}{l}\text { Risk of } \\
\text { undernutrition } \\
\text { by } \text { MUST }^{\S}\end{array}$} & Low & $133(90.5 \%)$ & $14(9.5 \%)$ & \\
\hline & Medium & 14 (93.3\%) & $1(6.7 \%)$ & \\
\hline & High & $8(100 \%)$ & 0 & 0.622 \\
\hline \multirow{2}{*}{$\begin{array}{l}\text { SNUBH-NST } \\
\text { high risk }\end{array}$} & No & 150 (90.9\%) & 15 (9.1\%) & \\
\hline & Yes & $5(100 \%)$ & 0 & 1.000 \\
\hline
\end{tabular}

We divided our patients into two groups according to the presence or absence of morbidity for identifying the association between the preoperative nutritional status and postoperative complications. Patients with Dindo grade I or severe were included to morbidity positive group and patients without any complication to morbidity negative group.

${ }^{*}$ Body mass index; ${ }^{\dagger}$ Total lymphocyte count; ${ }^{\dagger}$ Nutritional Risk Screening; ${ }^{\S}$ Malnutrition Universal Screening Tool; "Seoul National University Bundang Hospital Nutritional Screening Tool.
There was no postoperative mortality in our study. Although patients in the CRC group had more advanced cancer, there were no significant differences in postoperative morbidity between the two groups. The frequency of major complications, which were classified Calvien-Dindo 3 or higher as described above, was not significantly different between the two groups (Table 2). There were no preoperative nutritional factors related to postoperative morbidity (Table 3 ).

Fig. 1 shows the differences in nutritional status between the preoperative and postoperative periods in each group according to the SNUBH-NST, MUST, and NRS 2002. In both groups, nutritional status was lower in the postoperative period compared to the preoperative period.

At the time of discharge, serum albumin level was significantly higher in the CRC group than in the GC group $(\mathrm{P}=0.002)$. The high risk of undernutrition measured by MUST were significantly more in the GC group than in the CRC group (34.1\% vs. $16.5 \%, \mathrm{P}=0.007)$. Also, patients in the GC group more frequently experienced postoperative

\begin{tabular}{|c|c|c|c|c|}
\hline & & $\begin{array}{l}\text { Gastric } \\
\text { cancer } \\
(\mathrm{N}=85)\end{array}$ & $\begin{array}{c}\text { Colorectal } \\
\text { cancer } \\
(\mathrm{N}=85)\end{array}$ & P-value \\
\hline $\begin{array}{l}\text { Postoperative } \\
\text { hospital stay (days) }\end{array}$ & Mean \pm SD & $12.4 \pm 4.8$ & $9.5 \pm 6.7$ & 0.002 \\
\hline \multirow{2}{*}{$\mathrm{BMI}^{*}\left(\mathrm{~kg} / \mathrm{m}^{2}\right)$} & $<18.5$ & $2(2.4 \%)$ & $5(5.9 \%)$ & \\
\hline & $\geq 18.5$ & $83(97.6 \%)$ & 80 (94.1\%) & 0.443 \\
\hline \multirow[t]{2}{*}{$\mathrm{TLC}^{\dagger}$ (cells $/ \mathrm{mm}^{3}$ ) } & $<900$ & $15(17.6 \%)$ & $15(17.6 \%)$ & \\
\hline & $\geq 900$ & 70 (82.4\%) & $70(82.4 \%)$ & 1.000 \\
\hline \multirow[t]{2}{*}{ Albumin (g/dL) } & $<3.5$ & $48(57.1 \%)$ & $28(32.9 \%)$ & \\
\hline & $\geq 3.5$ & 36 (42.9\%) & $57(67.1 \%)$ & 0.002 \\
\hline \multirow[t]{2}{*}{ NRS $^{\dagger} 2002$ high risk } & No & $32(37.6 \%)$ & $33(38.8 \%)$ & \\
\hline & Yes & $53(62.4 \%)$ & $52(61.2 \%)$ & 1.000 \\
\hline \multirow{3}{*}{$\begin{array}{l}\text { Risk of undernutrition } \\
\text { by } \text { MUST }^{\S}\end{array}$} & Low & $32(37.6 \%)$ & $51(60.0 \%)$ & \\
\hline & Medium & $24(28.2 \%)$ & $20(23.5 \%)$ & \\
\hline & High & 29 (34.1\%) & $14(16.5 \%)$ & 0.007 \\
\hline \multirow{2}{*}{$\begin{array}{l}\text { SNUBH-NST" } \\
\text { high risk }\end{array}$} & No & $72(84.7 \%)$ & $73(85.9 \%)$ & \\
\hline & Yes & 13 (15.3\%) & $12(14.1 \%)$ & 1.000 \\
\hline \multirow{2}{*}{$\begin{array}{l}\text { Weight loss } \\
(5 \%)\end{array}$} & No & 38 (44.7\%) & $55(64.7 \%)$ & \\
\hline & Yes & $47(55.3 \%)$ & $30(35.3 \%)$ & 0.013 \\
\hline \multirow[t]{2}{*}{ Weight loss $(10 \%)$} & No & 62 (72.9\%) & 76 (89.4\%) & \\
\hline & Yes & $23(27.1 \%)$ & $9(10.6 \%)$ & 0.010 \\
\hline
\end{tabular}

*Body mass index; ${ }^{\dagger}$ Total lymphocyte count; ${ }^{\dagger}$ Nutritional Risk Screening; ${ }^{\S}$ Malnutrition Universal Screening Tool; "Seoul National University Bundang Hospital Nutritional Screening Tool. 


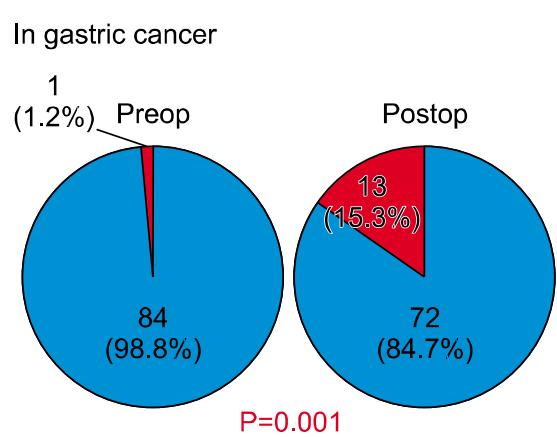

$<$ SNUBH-NST>

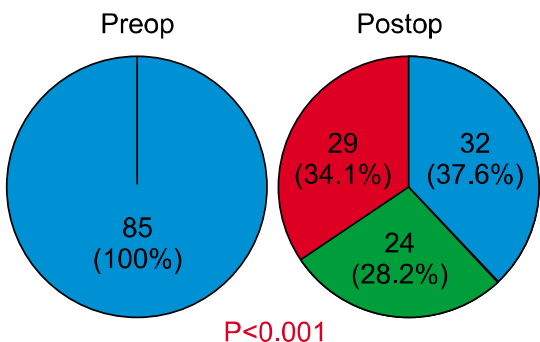

$<$ MUST>

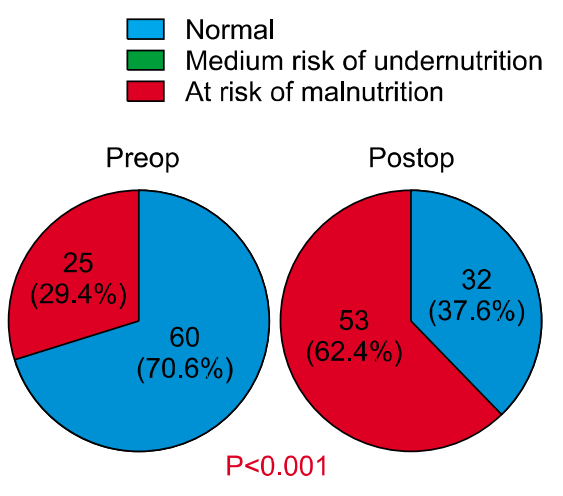

$<$ NRS 2002>

In colorectal cancer

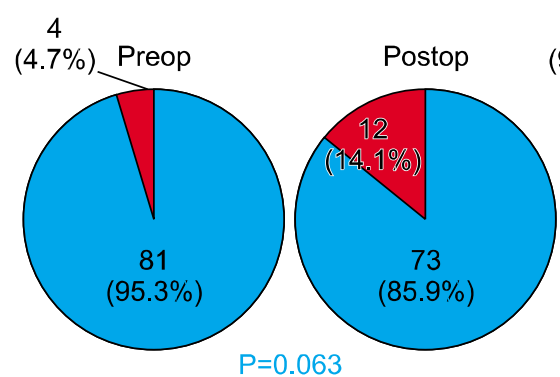

$<$ SNUBH-NST>

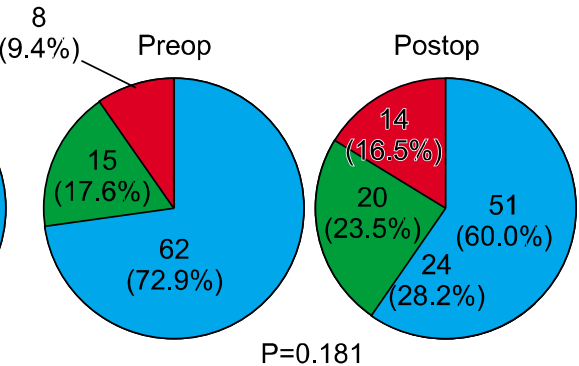

$<$ MUST>

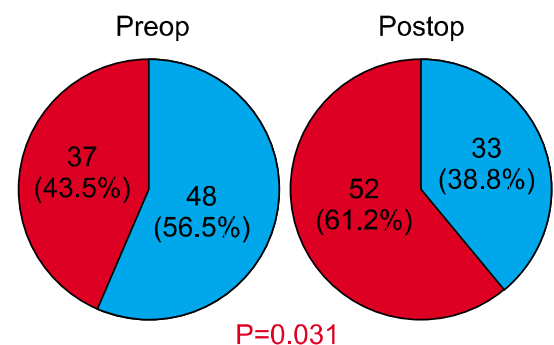

$<$ NRS 2002>

Fig. 1. Differences between preoperative and postoperative periods in the distribution of patients with risk of malnutrition according to the nutritional screening tools. In patients with gastric cancer, all three screening tools indicate significant impairment of the nutritional status after surgery. On the other hand, only NRS 2002 indicates significant impairment of nutritional status after colorectal cancer surgery. Although there were no significant differences, the number of patients at risk of malnutrition after surgery is higher according the SNUBH-NST and MUST.

SNUBH-NST = the Seoul National University Bundang Hospital Nutritional Screening Tool; MUST = the Malnutrition Universal Screening Tool; NRS = the Nutritional Risk Screening.

weight loss than those in the CRC group (Table 4).

The type of surgery did not affect postoperative morbidity. Also, the type of surgery was not related with postoperative BMI or postoperative serum albumin level. Total gastrectomy of the GC group was significantly associated with a risk of postoperative malnutrition according to MUST and marginally with a risk of postoperative malnutrition according to NRS 2002. Also, total gastrectomy was significantly related to postoperative weight loss in the GC group. Of the CRC group, proctectomy and total mesorectal excision with or without diverting stoma were marginally associated with postoperative weight loss (Table 5).

\section{DISCUSSION}

GC and CRC are the most common gastrointestinal tu- mors, and both of these are among the most common cancers in Korea.[13] A gastrointestinal tumor can cause problems related to nutrition in various ways. Passage disturbance by obstructive mass, tumor perforation, intestinal anastomosis after surgery, complications and sequelae after surgery, and the tumor itself are representative causes related to the risk of malnutrition. Specifically, the complications related to anastomosis such as leakages and strictures can require the patients to maintain NPO for a long time, which can result in malnutrition. In addition, the functional sequelae such as dysphagia or reflux after gastric surgery and frequent defecation or obstructive defecation after colorectal surgery can induce poor oral intake and result in malnutrition.

In the present study, we assessed patient postoperative nutritional status at the time of discharge. In both GC and CRC groups, postoperative nutritional status was worse 


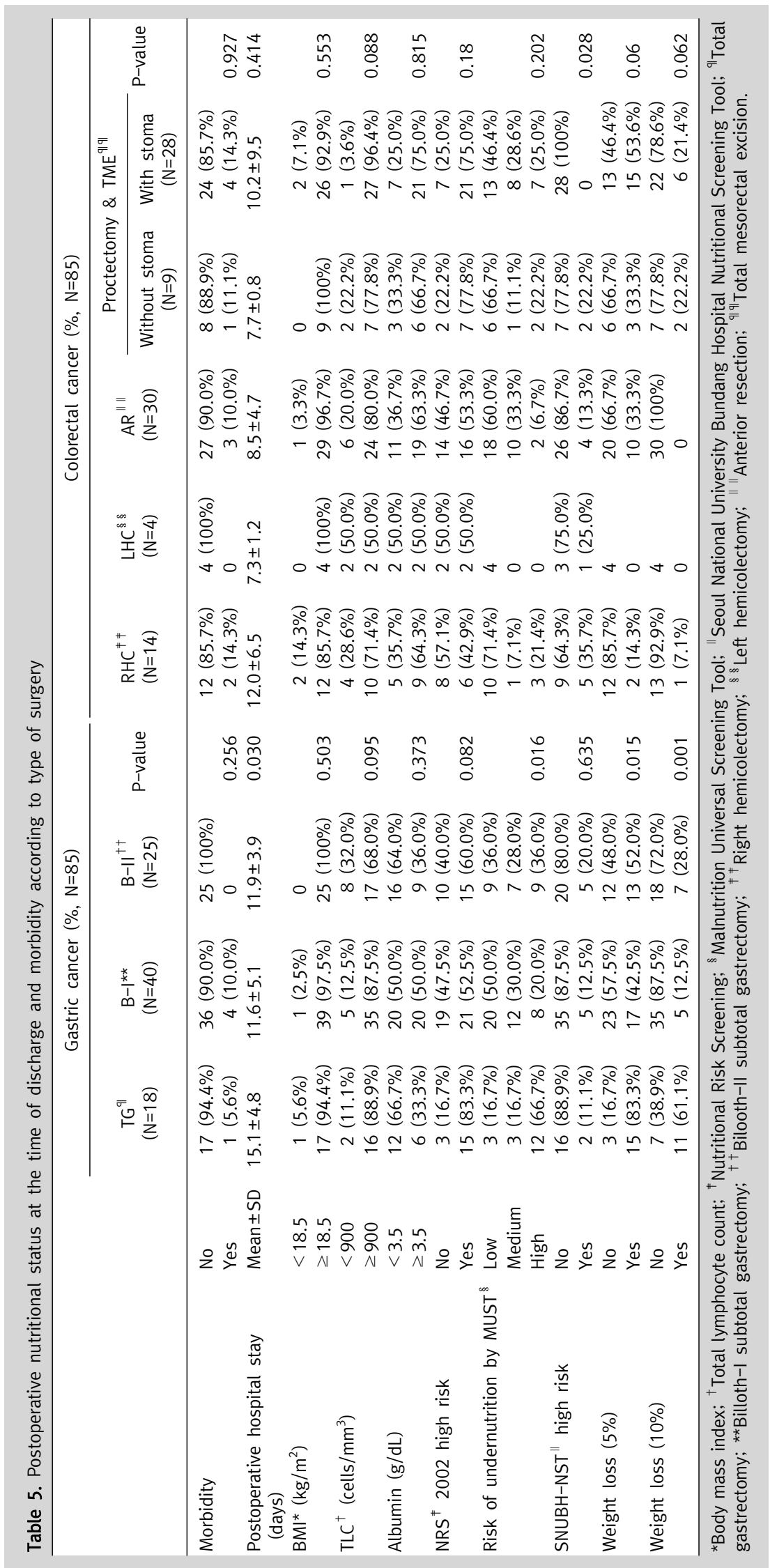


than the preoperative status (Fig. 1). In the recent surgical environment, cancer patients undergoing surgery have been treated using ERAS, which seeks to minimize surgical stress, maintain nutritional status, reduce complications, and optimize the rate of recovery.[14,15] In our study, we cared for most of our patients with standing orders that were similar to those of the ERAS program. Nevertheless, we found that postoperative nutritional status was significantly impaired in both the GC and CRC groups. From this result, it can be suggested that consecutive screening or assessment of patient nutritional status during the perioperative period should be performed to control and minimize the nutritional deficit during the postoperative period of gastrointestinal cancer patients and to provide the appropriate perioperative nutritional support in these patients.

Table 1 shows that the preoperative nutritional status in the CRC group was worse than in the GC group (higher mean age, lower serum albumin level, and higher risk according to the MUST). Table 4 shows opposing results after surgery. Patients in the GC group had a lower serum albumin level $(\mathrm{P}=0.005)$ and a higher risk according to the MUST $(\mathrm{P}=0.007)$ than those in the CRC group in the postoperative period. In addition, patients in the GC group lost more body weight than those in the CRC group after surgery. In one study analyzing the change between preoperative and postoperative nutritional status with a patient-generated subjective global assessment (PG-SGA), the proportion of severely malnourished GC patients increased significantly from $2.2 \%$ to $29.7 \%$ $(\mathrm{P}=0.006)$ after gastrectomy. On the other hand, among CRC patients, such changes were not significant.[16] These results demonstrate that old age, preoperative weight loss, open surgery, and GC were significant risk factors for postoperative malnutrition. The reason that patients in the GC group experienced greater weight loss in the postoperative period might be that resection of the stomach reduces reservoir function and can cause early fullness and smaller caloric intake.[16] Also, having looked back at our standing orders, a prolonged period of NPO in the GC group might be attributed to worse post- operative nutritional status. Although the effect of parenteral nutrition is not obvious, the development of special elements or materials for parenteral nutrition during the NPO period may be helpful for minimizing nutritional deficit and maintaining nutritional status. Besides parenteral nutrition during NPO, active supports with oral nutritional supplement after resumption of oral feeding may be one of solutions to prevent postoperative nutritional deficit for these patients who undergo radical surgery.

Generally, we performed radical surgery for GC and CRC. The type of surgery in both cancers usually depends on the tumor location. The types of surgery mainly applied to GC patients are subtotal and total gastrectomy and those to CRC are right hemicolectomy, left hemicolectomy, anterior resection, and low anterior resection with or without diverting stoma. Because the segment of resected intestine and the range of lymphadenectomy depend on the type of surgery, various nutritional conditions can result from the different surgeries. In the present study, the type of operation in the GC group significantly affected the postoperative risk of malnutrition according to the MUST and weight loss. Patients who underwent total gastrectomy had a higher risk of malnutrition according to the MUST ( $\mathrm{P}=0.016)$ and weight loss. Kosuga et al. [17] demonstrated that laparoscopic subtotal gastrectomy had a significant advantage over laparoscopic total gastrectomy with regard to the postoperative nutritional status and the incidence of postoperative severe reflux esophagitis. Also, some investigators demonstrated that the reason for the difference between total gastrectomy and subtotal gastrectomy was the physical functioning of the stomach, the changes in the role of the stomach, appetite loss, and eating restrictions scales.[18] Also, a marginally higher portion of patients (83.3\%) who underwent total gastrectomy had a higher risk of malnutrition according to the NRS $2002(\mathrm{P}=0.082)$. The NRS 2002 questionnaire included an item related to food intake. Therefore, we could consider the change of food intake before vs. after surgery. In the CRC group, although there were no statistically significant differences, proctectomy with TME regardless of diverting stoma was 
marginally associated with postoperative weight loss. In our report related with diverting stoma after rectal cancer surgery, we observed that stoma creation induced about 5\% weight loss in our rectal cancer patients.[19] In most patients with $\mathrm{CRC}$, diets might not change and nutritional status is stable. But, change in defecation including frequent defecation, watery diarrhea, and incontinence or obstructive defecation such as low anterior resection syndrome could affect the amount, frequency, and nature of the diet. Because of this association between defecation and oral intake, some patients who underwent surgery for CRC might have lost weight or been at risk for malnutrition. Actually, comparing Table 1 and Table 4, we can observe that the risk of malnutrition according to the MUST and NRS 2002 in patients with CRC was increased, with $35.3 \%$ of patients experiencing more than $5 \%$ weight loss after surgery. Specifically, 53.5\% of patients with diverting stoma after proctectomy experienced more than 5\% weight loss after surgery. More than $70 \%$ of patients with proctectomy, which might be closely related to defecation dysfunction or stoma, had a risk of malnutrition according to the NRS 2002 (Table 5). We think this finding is akin to the observed results in GC patients.

We could have allowed the patients in nutritionally compromised status to leave the hospital. However, a certain portion of patients who underwent curative resection needed adjuvant therapy. The postoperative nutritional status was closely related to the timing of starting adjuvant therapy. Although we could not analyze the relation between nutritional deficits and the application of adjuvant therapy, adjuvant therapy for some patients must have been postponed or not been applied due to their poor nutritional status. Therefore, we should recognize that the postoperative nutritional status in gastrointestinal cancer patients could be worse than that in the preoperative period and aim to improve the postoperative nutritional outcome in gastrointestinal cancer patients.

Our study had some limitations. Despite the use of propensity score matching, the small number of patients, retrospective nature, and some missing data might have biased the results of this study. Also, since this is a retrospective study, the exact scheduled time frame related to re-weighing, the exact volume status of patients at the time of postoperative nutritional screening, and the relationship between albumin level and other inflammatory parameters were not available for analysis. Especially, if the inflammatory parameters like C-reactive protein were presented in this study, it could be possible to clarify the change of nutritional risk after radical surgery. Because the results were short-term outcomes, we did not evaluate how poor nutritional status affected patient quality of life after discharge. We also did not identify the application rate of neoadjuvant or adjuvant therapy in both groups. However, to the best of our knowledge, the results of our study are the most comprehensive in regard to nutritional status after gastrointestinal cancer surgery. We expect that well-designed prospective study will be able to explain the change of nutritional status after gastrointestinal cancer surgery.

In conclusion, postoperative nutritional status tends to be significantly impaired in patients who undergo gastrointestinal cancer surgery. Despite the nutritional support during NPO after surgery, gastrointestinal cancer surgery can negatively affect postoperative nutritional status, especially after GC surgery. Therefore, consecutive assessments of nutritional status and appropriate nutritional support are necessary after surgery for appropriate treatment such as the introduction of timely oncological care.

\section{REFERENCES}

1. Thoresen L, Frykholm G, Lydersen S, Ulveland H, Baracos V, Prado CM, et al. Nutritional status, cachexia and survival in patients with advanced colorectal carcinoma. Different assessment criteria for nutritional status provide unequal results. Clin Nutr 2013;32:65-72.

2. Bosaeus I. Nutritional support in multimodal therapy for cancer cachexia. Support Care Cancer 2008;16:447-51.

3. Von Meyenfeldt MF, Meijerink WJ, Rouflart MM, Builmaassen MT, Soeters PB. Perioperative nutritional support: a randomised clinical trial. Clin Nutr 1992;11:180-6.

4. Meguid MM, Curtas MS, Meguid V, Campos AC. Effects of pre-operative TPN on surgical risk--preliminary status report. $\mathrm{Br}$ J Clin Pract Suppl 1988;63:53-8.

5. Bozzetti F, Gavazzi C, Miceli R, Rossi N, Mariani L, Cozzaglio $\mathrm{L}$, et al. Perioperative total parenteral nutrition in malnourished, gastrointestinal cancer patients: a randomized, clinical 
trial. JPEN J Parenter Enteral Nutr 2000;24:7-14.

6. Gustafsson UO, Ljungqvist $O$. Perioperative nutritional management in digestive tract surgery. Curr Opin Clin Nutr Metab Care 2011;14:504-9.

7. Arends J, Bachmann P, Baracos V, Barthelemy N, Bertz H, Bozzetti $F$, et al. ESPEN guidelines on nutrition in cancer patients. Clin Nutr 2017;36:11-48.

8. Dindo D, Demartines N, Clavien PA. Classification of surgical complications: a new proposal with evaluation in a cohort of 6336 patients and results of a survey. Ann Surg 2004;2 40:205-13.

9. Kondrup J, Allison SP, Elia M, Vellas B, Plauth M; Educational and Clinical Practice Committee, European Society of Parenteral and Enteral Nutrition (ESPEN). ESPEN guidelines for nutrition screening 2002. Clin Nutr 2003;22:415-21.

10. Kim SY, Yeom HS, Park YM, Chung SH, Shin AR, Han HS, et al. Comparison of tools for nutritional risk screening at hospital admission. J Korean Soc Parenter Enter Nutr 2009;2:6-12.

11. Yang $Y$, Gao P, Song $Y$, Sun J, Chen X, Zhao J, et al. The prognostic nutritional index is a predictive indicator of prognosis and postoperative complications in gastric cancer: a meta-analysis. Eur J Surg Oncol 2016;42:1176-82.

12. Onodera T, Goseki N, Kosaki G. Prognostic nutritional index in gastrointestinal surgery of malnourished cancer patients. Nihon Geka Gakkai Zasshi 1984;85:1001-5.
13. Jung KW, Won YJ, Oh CM, Kong HJ, Lee DH, Lee KH. Cancer statistics in Korea: incidence, mortality, survival, and prevalence in 2014. Cancer Res Treat 2017;49:292-305.

14. Gustafsson UO, Scott MJ, Schwenk W, Demartines N, Roulin $D$, Francis N, et al. Guidelines for perioperative care in elective colonic surgery: enhanced recovery after surgery (ERAS $\circledast$ ) Society recommendations. Clin Nutr 2012;31:783-800.

15. Makuuchi R, Sugisawa N, Kaji S, Hikage M, Tokunaga M, Tanizawa $\mathrm{Y}$, et al. Enhanced recovery after surgery for gastric cancer and an assessment of preoperative carbohydrate loading. Eur J Surg Oncol 2017; 43:210-7.

16. Shim H, Cheong JH, Lee KY, Lee H, Lee JG, Noh SH. Perioperative nutritional status changes in gastrointestinal cancer patients. Yonsei Med J 2013;54:1370-6.

17. Kosuga T, Hiki N, Nunobe S, Noma H, Honda M, Tanimura S, et al. Feasibility and nutritional impact of laparoscopy-assisted subtotal gastrectomy for early gastric cancer in the upper stomach. Ann Surg Oncol 2014;21:2028-35.

18. Kobayashi D, Kodera Y, Fujiwara M, Koike M, Nakayama G, Nakao A. Assessment of quality of life after gastrectomy using EORTC QLQ-C30 and STO22. World J Surg 2011;35:357-64.

19. Kye BH, Kim HJ, Kim JG, Cho HM. The nutritional impact of diverting stoma-related complications in elderly rectal cancer patients. Int J Colorectal Dis 2013;28:1393-400. 